\title{
Clinicopathological Analysis of Medullary Ray Injury in 1-Year Protocol Paediatric Renal Allograft Biopsies
}

\author{
Junya Hashimoto ${ }^{a}$ Hideyo Oguchi $^{\mathrm{a}}$ Tetuo Mikami ${ }^{\mathrm{b}}$ Yuko Hamasaki ${ }^{\mathrm{a}}$ \\ Masaki Muramatsu $^{\text {a }}$ Yutaka Yamaguchi $^{c}$ Ken Sakai $^{a}$ \\ aDepartment of Nephrology, Toho University Faculty of Medicine, Tokyo, Japan; bepartment of Pathology, Toho \\ University Faculty of Medicine, Tokyo, Japan; 'Yamaguchi's Pathology Laboratory, Chiba, Japan
}

\section{Keywords \\ Calcineurin inhibitor nephrotoxicity - Graft function . Medullary ray injury . Paediatric renal transplantation . Protocol biopsy}

\begin{abstract}
Aim: Medullary ray injury was recently reported in renal transplant biopsies. This study was performed to clarify the clinicopathological features of medullary ray injury in paediatric living renal transplant recipients. Methods: Paediatric recipients who completed a 5 -year follow-up after living renal transplantation were enroled. We evaluated the clinical and pathological parameters of the presence or absence of medullary ray injury in their 1-year protocol biopsies. $\mathbf{R e}$ sults: Of 48 1-year protocol biopsies, 18 (37.5\%) showed histological evidence of medullary ray injury. The 48 paediatric recipients were classified as those with medullary ray injury ( $n=18$; MRI-1Y [+] group) and those without medullary ray injury ( $n=30$; MRI-1Y [-] group) in the 1-year protocol biopsies. The prevalence of histological evidence of calcineurin inhibitor (CNI) nephrotoxicity, chronic obstruction or reflux nephropathy, and imaging findings of vesicoureteral reflux was $66.7,22.2$, and $7.7 \%$ in the MRI-1Y (+) group and 33.3, 13.3 , and $15.4 \%$ in the MRI-1Y (-) group, respectively. Only
\end{abstract}

the prevalence of $\mathrm{CNI}$ nephrotoxicity was significantly different between the 2 groups. There was no significant difference in the mean estimated glomerular filtration rate at 1,3, or 5 years after transplantation between the 2 groups. Conclusion: In total, $37.5 \%$ of 1-year protocol biopsies showed histological evidence of medullary ray injury. This finding suggests that CNI nephrotoxicity might be the main contributor to medullary ray injury in 1-year protocol biopsies. The presence of medullary ray injury had little influence on renal function, at least during the first 5 years after transplantation.

(c) 2020 The Author(s)

Published by S. Karger AG, Basel

\section{Introduction}

A previous study showed that administration of cyclosporine resulted in a striped pattern of fibrosis involving the medullary ray and outer medulla in a rat model [1]. In another study, a striped pattern of fibrosis was observed in kidney transplant recipients who received both FK506 and cyclosporine [2]. Kobayashi et al. [3] recently defined tubular atrophy and interstitial fibrosis restricted to the medullary ray as "medullary ray injury (MRI)" and showed that the causes of MRI can be categorized as cal- 
Fig. 1. Representative figure of MRI in paediatric renal transplant recipients. Striped fibrosis and infiltrative inflammation were found in the medullary rays (Masson's trichrome stain, $\times 100)$. MRI, medullary ray injury.

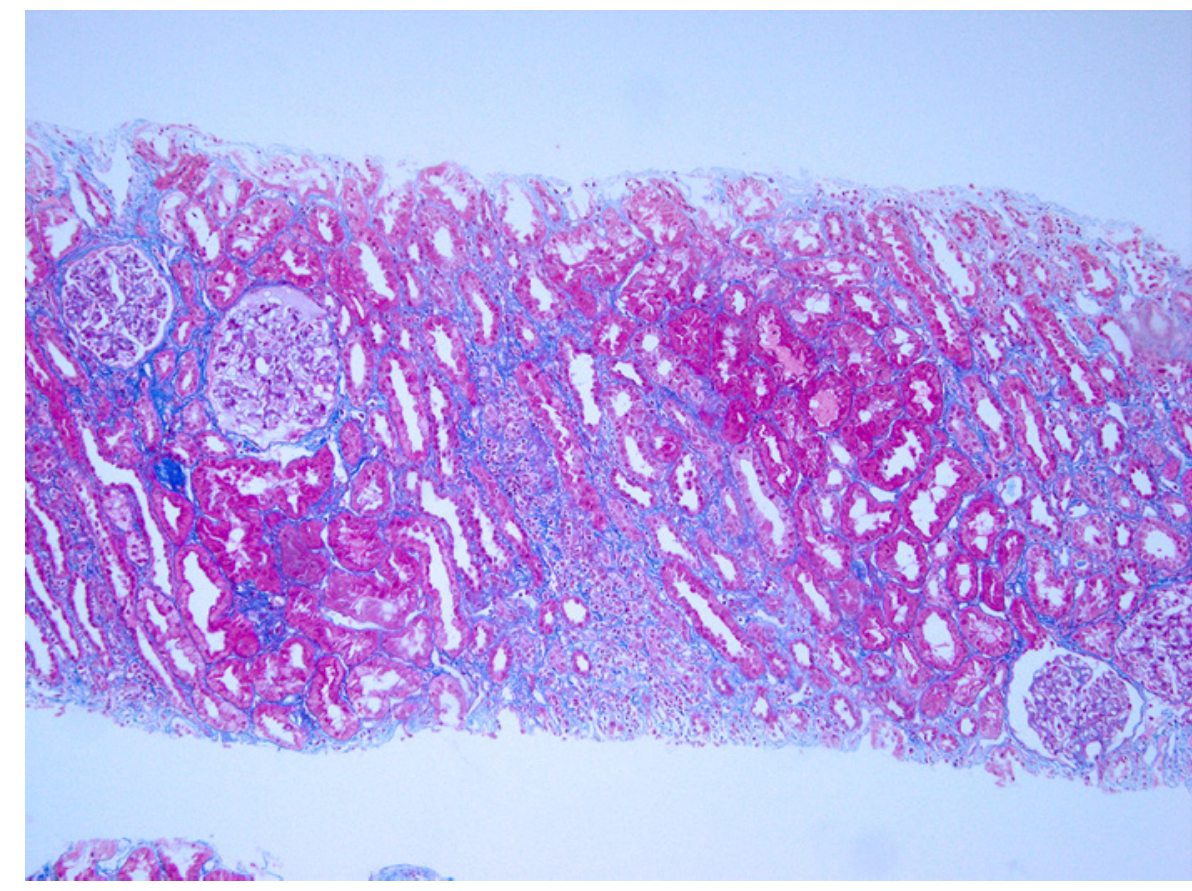

cineurin inhibitor (CNI) nephrotoxicity, chronic obstruction of the urinary system, and pyelonephritis, among other causes.

Our routine pathological observations have shown that MRI is often found in paediatric early allograft protocol biopsy (Fig. 1). However, the long-term prognosis after paediatric kidney transplantation was recently reported to have improved [4]. Congenital anomalies of the kidney and urinary tract, including reflux or obstructive urinary tract disease, are the most common underlying diseases resulting in the need for paediatric kidney transplantation $[5,6]$. The clinicopathological significance of MRI, especially in paediatric allografts, has been rarely reported to date. The present study was performed to clarify the prevalence, cause, and clinicopathological prognosis of MRI using paediatric renal transplant protocol biopsy specimens.

\section{Materials and Methods}

\section{Patients}

We enroled 48 paediatric recipients who underwent living-donor renal transplantation in our department from March 2009 to March 2014. They completed a 5-year follow-up after transplantation. The age of the enroled recipients was limited to $<16$ years at the time of transplantation. We retrospectively analysed data from the patients' medical records. The body size mismatch between the recipient and donor at transplantation was evaluated by the ratio of the recipient's body surface area (BSA) to the donor's BSA. In our department, as many paediatric transplant patients as possible undergo routine voiding cystourethrography (VCUG) by 1-year protocol biopsy to evaluate the prevalence of vesicoureteral reflux (VUR) to the transplanted kidney. We also assessed the prevalence of VUR in the present study.

\section{Pathological Analysis}

The renal allograft biopsy samples were pathologically evaluated according to the criteria of the Banff classifications [7-9]. MRI was defined as the presence of 3 or more areas of tubular atrophy and interstitial fibrosis localized to the medullary ray in the renal allograft biopsy samples. We evaluated the recipients' clinical and pathological parameters based on the presence or absence of MRI in their 1-year protocol biopsies. The patients were divided into 2 groups according to the presence or absence of MRI in their 1-year protocol biopsy: those with MRI $(n=18$; MRI-1Y $[+]$ group) and those without MRI ( $n=30$; MRI-1Y [-] group). We defined histological evidence of CNI nephrotoxicity, chronic or reflux nephropathy, and pyelonephritis according to a previous report as follows: CNI nephrotoxicity, either an arteriolar hyaline lesion (aah score of $\geq 1$ ) or isometric vacuolization of tubular cells; chronic obstruction or reflux nephropathy, Tamm-Horsfall protein casts with extravasation into the interstitium; and pyelonephritis, intratubular and peritubular predominant neutrophil infiltration with thyroidization [3]. We also examined the prevalence of acute $\mathrm{T}$ cell-mediated rejection or antibody-mediated rejection and BK polyomavirus nephropathy based on pathological diagnosis.

Arteriosclerosis of medium-sized arteries in baseline biopsies at transplantation or in biopsies of the renal allograft at evaluation of tacrolimus nephrotoxicity reportedly affects the presence of tacrolimus nephrotoxicity $[10,11]$. We examined the prevalence of interlobular arteriosclerosis in 1-hour renal allograft biopsies as the baseline according to the above-mentioned previous report [11]. 
Table 1. Clinical characteristics of 1-year protocol biopsy

\begin{tabular}{lc}
\hline & $n=48$ \\
\hline Recipient sex (male/female) & $29 / 19$ \\
Recipient age at transplantation, years & $7.5 \pm 4.0$ \\
Recipient height at transplantation, cm & $109.6 \pm 26.2$ \\
Recipient body weight at transplantation, kg & $20.1 \pm 12.1$ \\
Original disease: CAKUT & $27(56.3)$ \\
ABO-incompatible transplantation & $7(14.6)$ \\
Donor sex (male/female) & $21 / 27$ \\
Donor age at transplantation, years & $40.1 \pm 6.6$ \\
Donor eGFR at transplantation, mL/min/1.73 m m $^{2}$ & $79.9 \pm 16.0$ \\
R-BSA & $0.77 \pm 0.32$ \\
D-BSA & $1.66 \pm 0.17$ \\
R-BSA/D-BSA & $0.47 \pm 0.19$ \\
Arteriosclerosis in 1-hour renal allograft biopsy & $16(33.3)$ \\
Recipients performed VCUG by 1-year & $39(81.3)$ \\
$\quad$ protocol biopsy & $5 / 39(12.8)$ \\
VUR to the transplanted kidney & $0(0.0)$ \\
Graft loss during the observation period & $0(0.0)$ \\
Recipient death during the observation period & \\
\hline
\end{tabular}

Data are presented as $n$, mean \pm standard deviation, or $n(\%)$. CAKUT, congenital anomalies of the kidney and urinary tract; eGFR, estimated glomerular filtration rate; R, recipient; D, donor; BSA, body surface area; VCUG, voiding cystourethrography; VUR, vesicoureteral reflux.

Additionally, we compared the change in the prevalence of MRI and the changes in the ci $+\mathrm{ct}$ and aah scores in the 1-, 3-, and 5 -year protocol biopsies. Patients whose protocol renal biopsy could not be performed 3 or 5 years after transplantation were excluded from these statistical analyses as needed.

\section{Allograft Function}

We compared the changes in the transplanted renal function between the 2 study groups at 1, 3, and 5 years after transplantation. We adopted the estimated glomerular filtration rate (eGFR) as an index of renal graft function. The eGFR was calculated using a Cr-based equation for Japanese children and adolescents aged 19 years $[12,13]$ and Japanese adults aged $\geq 19$ years [14].

\section{Statistical Analysis}

Data are expressed as mean \pm standard deviation. Student's $t$ test and the Wilcoxon rank sum test were used to compare continuous variables between 2 unpaired groups. A paired $t$ test was used to compare continuous variables between 2 paired groups. The $\chi^{2}$ test was used to compare categorical variables between 2 paired groups. Factors related to MRI formation were analysed by univariate and multivariate approaches. The results are presented as the odds ratio (OR) and $95 \%$ confidence interval (CI), and a $p$ value of $<0.05$ was considered statistically significant. When conducting the multivariate analysis of factors affecting the formation of MRI, factors that were implicated in previous reports were selected as explanatory variables $[3,15]$.

Medullary Ray Injury in Paediatric

Recipients

\section{Results}

\section{Clinical Background Characteristics and Pathological Findings}

The patients' clinical characteristics are shown in Table 1 . The patient population comprised 29 male and 19 female recipients. Their mean age, height, and body weight at transplantation were $7.5 \pm 4.0$ years, $109.6 \pm$ $26.2 \mathrm{~cm}$, and $20.1 \pm 12.1 \mathrm{~kg}$, respectively. The original disease was a congenital anomaly of the kidney and urinary tract in 27 patients. ABO-incompatible transplantation was performed in 7 patients (14.6\%). The donors comprised 21 men and 27 women with a mean age of $40.1 \pm$ 6.6 years. The mean BSA of the recipients was $0.77 \pm 0.32$ $\mathrm{m}^{2}$, and the mean BSA of the donors was $1.66 \pm 0.17 \mathrm{~m}^{2}$. The mean ratio of the recipients' to donors' BSA was 0.47 \pm 0.19 . No recipient death or graft loss occurred during the observation period.

\section{Comparison of Background Characteristics of \\ MRI-1Y (+) and (-) Groups}

The background characteristics of the MRI-1Y (+) group and MRI-1Y (-) group are shown in Table 2. No significant differences were found in the mean age at the 1-year protocol biopsy, prevalence of recipient sex, mean recipient body weight at transplantation, prevalence of congenital anomalies of the kidney and urinary tract as the original disease, prevalence of $\mathrm{ABO}$-incompatible transplantation, prevalence of arteriosclerosis in the 1-hour renal allograft biopsy, or mean eGFR at the 1-year protocol biopsy between the 2 groups. The patients were divided into the tacrolimus group $(n=26)$ and cyclosporine group $(n=22)$ according to the type of CNI used at 1 year after transplantation, and the trough blood concentrations of these CNIs in the MRI-1Y $(+) /(-)$ group were not significantly different. By 1 year after transplantation, VCUG had been performed in 39 of 48 patients (81.3\%), and 5 of these 39 patients (12.8\%) had VUR. The prevalence of VUR was 1 of 13 patients $(7.7 \%)$ in the MRI-1Y (+) group and 4 of 26 patients (15.4\%) in the MRI-1Y (-) group, with no significant difference between them. The ci + ct score was significantly higher in the MRI-1Y (+) group, but the aah score was not different between the 2 groups. The MRI-1Y (+) group had a significantly higher frequency of CNI nephrotoxicity. There was no significant difference in the prevalence of chronic obstruction or reflux nephropathy. There were no cases of pyelonephritis in either group. No significant differences were also found in the prevalence of acute T cellmediated rejection or antibody-mediated rejection by 
Table 2. Comparison of clinical backgrounds and pathological findings of MRI-1Y (+) group and MRI-1Y (-) group

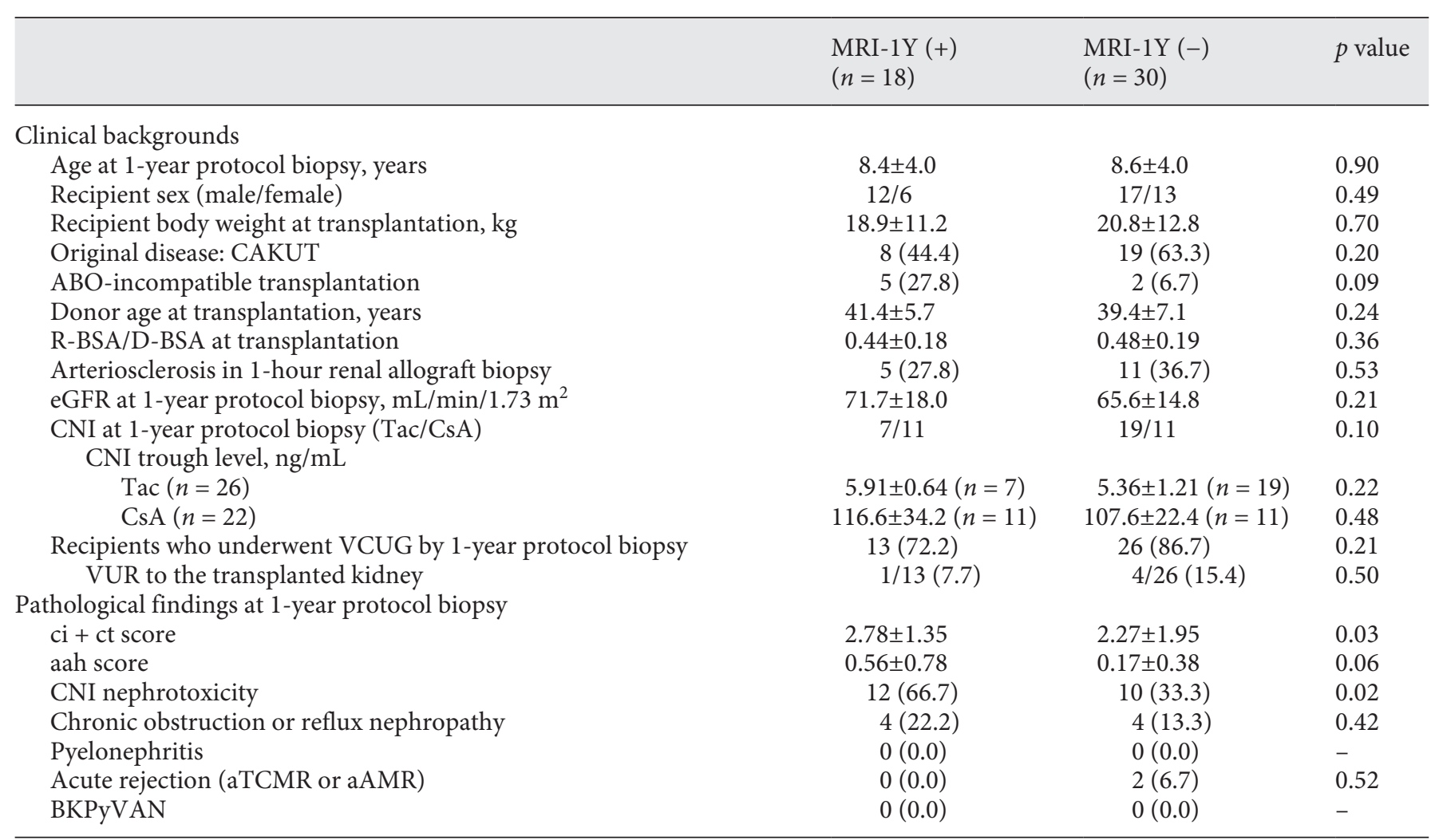

Data are presented as $n$, mean \pm standard deviation, or $n(\%)$. MRI-1Y (+), patients with medullary ray injury; MRI-1Y $(-)$, patients without medullary ray injury; CAKUT, congenital anomalies of the kidney and urinary tract; R, recipient; D, donor; BSA, body surface area; eGFR, estimated glomerular filtration rate; CNI, calcineurin inhibitor; Tac, tacrolimus; CsA, cyclosporine; VCUG, voiding cystourethrography; VUR, vesicoureteral reflux; aTCMR, acute T cell-mediated rejection; aAMR, acute antibody-mediated rejection; BKPyVAN, BK polyomavirus nephropathy.

Table 3. Risk factors for MRI in 1-year protocol biopsy by univariate and multivariate analyses

\begin{tabular}{|c|c|c|c|c|c|c|}
\hline Factor & OR & $95 \% \mathrm{CI}$ & $p$ value & OR & $95 \% \mathrm{CI}$ & $p$ value \\
\hline Recipient body weight at transplantation $(\mathrm{kg})$ & 0.99 & $0.94-1.04$ & 0.60 & - & - & - \\
\hline Recipient sex (male) & 1.53 & $0.45-5.17$ & 0.49 & - & - & - \\
\hline Original disease: CAKUT & 0.46 & $0.14-1.52$ & 0.20 & - & - & - \\
\hline R-BSA/D-BSA at transplantation & 0.37 & $0.03-4.29$ & 0.43 & - & - & - \\
\hline CNI at 1-year protocol biopsy (Tac) & 0.36 & $0.11-1.23$ & 0.10 & - & - & - \\
\hline CNI nephrotoxicity & 4.00 & $1.15-13.8$ & 0.03 & 3.85 & $1.10-13.4$ & 0.03 \\
\hline Chronic obstruction or reflux nephropathy & 1.86 & $0.40-8.58$ & 0.43 & 1.49 & $0.30-7.54$ & 0.63 \\
\hline Pyelonephritis & - & - & - & - & - & - \\
\hline Acute rejection (aTCMR or aAMR) & - & - & - & - & - & - \\
\hline
\end{tabular}

OR, odds ratio; CI, confidence interval; CAKUT, congenital anomalies of the kidney and urinary tract; R, recipient; D, donor; BSA, body surface area; CNI, calcineurin inhibitor; Tac, tacrolimus; aTCMR, acute T cell-mediated rejection; aAMR, acute antibody-mediated rejection; BKPyVAN, BK polyomavirus nephropathy. 


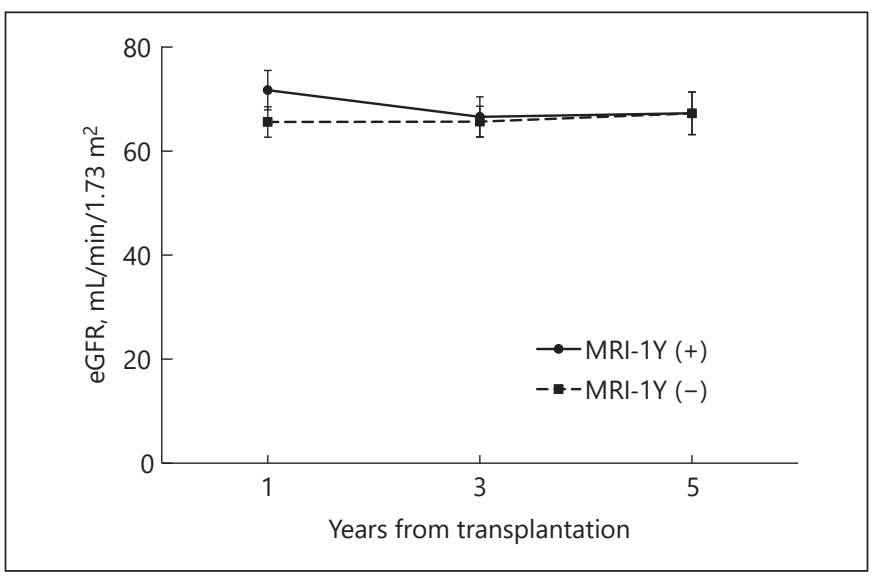

Fig. 2. Comparison of the mean eGFR in the MRI-1Y $(+) /(-)$ groups at 1,3 , and 5 years after transplantation. The error bars show the standard errors. eGFR, estimated glomerular filtration rate; MRI, medullary ray injury; MRI-1Y (+), patients with medullary ray injury; MRI-1Y (-), patients without medullary ray injury.

1-year protocol biopsy between the 2 groups. There were no cases of BK polyomavirus nephropathy in either group.

\section{Risk Factors for MRI Formation}

We evaluated the risk factors for MRI in the 1-year protocol biopsy via univariate and multivariate analyses (Table 3). The univariate analysis suggested that CNI nephrotoxicity (OR, $4.00 ; 95 \% \mathrm{CI}, 1.15-13.8 ; p=0.03$ ) could be a risk factor significantly associated with formation of MRI. The multivariate analysis also suggested that CNI nephrotoxicity could be a risk factor significantly associated with formation of MRI (OR, 3.85; 95\% CI, 1.10$13.4 ; p=0.03)$.

\section{Renal Function up to 5 Years after Transplantation}

We compared the mean eGFR in the MRI-1Y $(+) /(-)$ groups at 1, 3, and 5 years after transplantation (Fig. 2). The mean eGFR in the MRI-1Y (+) group at 1,3 , and 5 years after transplantation was $71.7 \pm 18.0,66.6 \pm 20.7$, and $67.3 \pm 19.8 \mathrm{~mL} / \mathrm{min} / 1.73 \mathrm{~m}^{2}$, respectively. The mean eGFR in the MRI-1Y (-) group at 1,3 , and 5 years after transplantation was $65.6 \pm 14.8,65.6 \pm 13.0$, and $61.9 \pm$ $15.8 \mathrm{~mL} / \mathrm{min} / 1.73 \mathrm{~m}^{2}$, respectively. There was no significant difference in the mean eGFR at each of these times between the 2 groups. In the MRI-1Y (+) group, the eGFR at 5 years was not significantly different from that at 1 year $(p=0.16)$.

Medullary Ray Injury in Paediatric Recipients

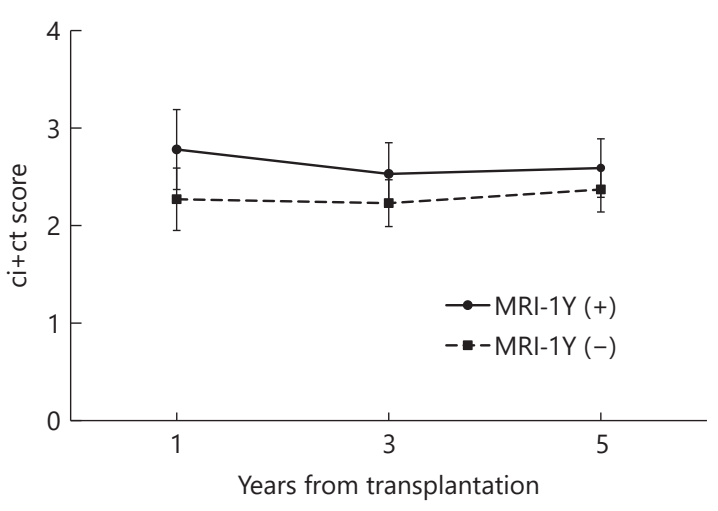

Fig. 3. Comparison of the mean ci + ct scores in the MRI-1Y $(+) /$ (-) groups. The error bars show the standard errors. MRI, medullary ray injury; MRI-1Y (+), patients with medullary ray injury; MRI-1Y (-), patients without medullary ray injury.

\section{Change in ci + ct Score up to 5 years after}

Transplantation

Of the 18 recipients in the MRI-1Y (+) group, 17 regularly underwent a protocol renal biopsy up to 5 years after transplantation, and only 1 patient in the MRI-1Y (+) group was excluded from the statistical analysis of the pathological findings at the 3 - and 5-year protocol biopsies. The changes in the $\mathrm{ci}+\mathrm{ct}$ score in the MRI-1Y $(+) /$ $(-)$ groups are shown in Figure 3 . The mean ci + ct score in the MRI-1Y $(+) /(-)$ groups at 1,3 , and 5 years after transplantation was $2.78 \pm 1.35 / 2.27 \pm 1.95,2.53 \pm$ $1.46 / 2.23 \pm 1.22$, and $2.59 \pm 1.12 / 2.37 \pm 1.30$, respectively. There was a significant difference in the mean ci $+\mathrm{ct}$ score between the 2 groups only at 1 year after transplantation $(p=0.03)$. In the MRI-1Y (+) group, the mean ci + ct score at 1 year was not different from that at 5 years $(p=0.76)$.

\section{Change in Prevalence of MRI Lesions up to 5 Years after Transplantation}

The prevalence of MRI lesions in our patients at 1,3 , and 5 years after transplantation was $37.5,36.2$, and $36.2 \%$, respectively. There was almost no change in the prevalence of MRI lesions up to 5 years after transplantation.

Comparison of aah Score in Patients with MRI at 1, 3, and 5 Years

The mean aah score in the patients with MRI at 1,3 , and 5 years after transplantation was $0.56 \pm 0.78(n=18)$, $0.53 \pm 0.80(n=17)$, and $0.71 \pm 0.77(n=17)$, respective- 
ly. No significant differences were found in the comparison of these scores between 1 and 3 years or between 1 and 5 years ( $p=0.89$ and $p=0.43$, respectively).

\section{Discussion}

There are few reports of the clinicopathological characteristics of MRI in paediatric renal transplant recipients. The purpose of our study was to investigate the aetiology and short-term prognosis of the 1-year protocol biopsy of paediatric recipients.

In the present study, the main cause of MRI in paediatric recipients was considered to be CNI nephrotoxicity. Kobayashi et al. [3] reported that obstructive or reflux nephropathy caused $36.1 \%$ of MRI and that VUR was proven in 6 of 7 recipients who underwent voiding cystography; however, our data showed that obstructive/reflux nephropathy seemed to be less involved in MRI formation. Akioka et al. [16] reported that VUR was proven in 8 of 10 paediatric renal transplant recipients with histological evidence of Tamm-Horsfall protein deposition to the interstitium. In the present study, VUR to the transplanted kidney was proven in only $1(7.7 \%)$ of 13 patients who underwent VCUG; therefore, histological evidence of obstructive/reflux nephropathy may not be as prominent as in previous reports [3].

A previous study showed that the blood flow of the renal artery after paediatric renal transplantation from an adult donor was lower than the blood flow of the donor before transplantation [17]. Another study showed that a small BSA in paediatric kidney recipients was associated with progressive tubulointerstitial damage [18]. Murray et al. [19] demonstrated that cyclosporine had a vasospasm effect in rat models. In our data, the CNI trough was not significantly higher in the MRI-1Y (+) group than in the MRI-1Y (-) group. The body size of the paediatric recipients in the present study was much smaller than that of the donors, and the average BSA of the recipients was about $50 \%$ that of the donors. There was no statistically significant difference in the ratio of the recipient to donor BSA between the MRI-1Y (+) and (-) groups. However, the ratio of the recipient to donor BSA was slightly lower in the MRI-1Y (+) group. Because graft blood flow reportedly decreases after paediatric kidney transplantation [17], we speculate that MRI caused by CNI nephrotoxicity may readily occur in paediatric renal allografts.

Niikura et al. [15] recently reported that the 3-year graft function was not significantly worse in recipients who had CNI-induced MRI at 3 months than in those who had no MRI at 3 months, and the 1 -year ci + ct scores were not worse than those from the baseline biopsies in the CNI-induced MRI group. In the present study, CNI nephrotoxicity may have been the main contributor to MRI in the 1-year protocol biopsies, and the graft function and the interstitial fibrosis and tubular atrophy score at 3 and 5 years were not significantly different between the 2 groups.

In this study, the prevalence of MRI at 1, 3, and 5 years after transplantation was almost constant. Additionally, there was no significant difference in the average aah score that could be associated with CNI toxicity in the MRI-1Y (+) group at 1, 3, and 5 years after transplantation. These data are consistent with the hypothesis that CNI toxicity is involved in MRI formation of transplanted kidneys in paediatric renal transplant recipients.

The main limitation of this study is that the results are limited to the clinicopathological course up to 5 years after transplantation only. Therefore, to elucidate the influence of MRI on the long-term prognosis, the clinical course of paediatric renal transplant recipients should be followed up for a longer period in future studies.

In conclusion, $37.5 \%$ of 1 -year protocol biopsies showed histological evidence of MRI. The data suggest that CNI nephrotoxicity was the main contributor to MRI in the 1-year protocol biopsies. The presence of MRI had little influence on renal function at least during the first 5 years after transplantation.

\section{Acknowledgements}

We are grateful to the kidney transplantation team in Department of Nephrology, Toho University Faculty of Medicine for contribution to planning or analysis or execution of this study. We are grateful to Kazutoshi Shibuya in Department of Surgical Pathology, Toho University Faculty of Medicine for contribution to managing the preparation of allograft biopsies. We also thank Angela Morben, DVM, ELS, from Edanz Group (www.edanzediting. com/ac), for editing a draft of this manuscript.

\section{Statement of Ethics}

This study was conducted in accordance with the principles contained within the Declaration of Helsinki and the ethical guidelines for epidemiological studies issued by the Ministry of Health, Labour and Welfare, Japan. The study was also approved by the ethics committee of Toho University Omori Medical Centre (ID: M19221 19059).
Hashimoto/Oguchi/Mikami/Hamasaki/ Muramatsu/Yamaguchi/Sakai 


\section{Conflict of Interest Statement}

The authors have no conflict of interest to declare.

\section{Author Contributions}

J.H. designed this study and collected the clinical data and wrote the paper. H.O. designed this study, performed pathological evaluation, and wrote the paper. T.M. contributed to pathological evaluation and drafting the work. Y.H., M.M., and K.S. contributed the design of the work and drafting the work. Y.Y. contributed to pathological evaluation and drafting the work. K.S. contributed to design of this study and drafting the work.

\section{References}

1 Rosen S, Greenfeld Z, Brezis M. Chronic cyclosporine-induced nephropathy in the rat. A medullary ray and inner stripe injury. Transplantation. 1990;49(2):445-52.

2 Randhawa PS, Shapiro R, Jordan ML, Starzl TE, Demetris AJ. The histopathological changes associated with allograft rejection and drug toxicity in renal transplant recipients maintained on FK506. Clinical significance and comparison with cyclosporine. Am J Surg Pathol. 1993;17(1):60-8.

3 Kobayashi A, Yamamoto I, Ito S, Akioka Y, Yamamoto H, Teraoka S, et al. Medullary ray injury in renal allografts. Pathol Int. 2010; 60(11):744-9.

4 Aoki Y, Hamasaki Y, Satoh H, Matsui Z, Muramatsu M, Hamada R, et al. Long-term outcomes of pediatric kidney transplantation: a single-center experience over the past 34 years in Japan. Int J Urol.2020 Feb;27(2):17278.

5 Garcia CD, Bittencourt VB, Tumelero A, Antonello JS, Moura DM, Vitola SP, et al. 300 pediatric renal transplantations: a single-center experience. Transplant Proc. 2006;38(10): 3454-5.

6 McEnery PT, Stablein DM, Arbus G, Tejani A. Renal transplantation in children. A report of the North American pediatric renal transplant cooperative study. N Engl J Med. Jun 25 1992;326(26):1727-32.
7 Racusen LC, Solez K, Colvin RB, Bonsib SM, Castro MC, Cavallo T, et al. The Banff 97 working classification of renal allograft pathology. Kidney Int. 1999;55(2):713-23.

8 Solez K, Colvin RB, Racusen LC, Haas M, Sis $\mathrm{B}$, Mengel M, et al. Banff 07 classification of renal allograft pathology: updates and future directions. Am J Transplant. 2008;8(4):75360.

9 Haas M, Sis B, Racusen LC, Solez K, Glotz D, Colvin RB, et al. Banff 2013 meeting report: inclusion of c4d-negative antibody-mediated rejection and antibody-associated arterial lesions. Am J Transplant. 2014;14(2):272-83.

10 Shimizu T, Ishida H, Shirakawa H, Omoto K, Tanabe K, Yamaguchi Y. Clinical and histological analysis of chronic tacrolimus nephrotoxicity in renal allografts. Transplant Proc. 2008;40(7):2370-2.

11 Yagisawa T, Omoto K, Shimizu T, Ishida $H$, Tanabe K. Arteriosclerosis in zero-time biopsy is a risk factor for tacrolimus-induced chronic nephrotoxicity. Nephrology. 2015; 20(Suppl 2):51-7.

12 Uemura O, Nagai T, Ishikura K, Ito S, Hataya $\mathrm{H}$, Gotoh Y, et al. Creatinine-based equation to estimate the glomerular filtration rate in Japanese children and adolescents with chronic kidney disease. Clin Exp Nephrol. 2014;18(4):626-33.

13 Uemura O, Ishikura K, Gotoh Y, Honda M. Creatinine-based estimated glomerular filtration rate for children younger than 2 years. Clin Exp Nephrol. 2018;22(2):483-4.
14 Matsuo S, Imai E, Horio M, Yasuda Y, Tomita K, Nitta K, et al. Revised equations for estimated GFR from serum creatinine in Japan. Am J Kidney Dis. 2009;53(6):982-92.

15 Niikura T, Kobayashi A, Kawabe M, Katsuma A, Yamakawa T, Katsumata H, et al. Clinicopathologic impact of early medullary ray injury in patients following kidney transplantation. Transplant Proc. 2017;49(1):78-83.

16 Akioka Y, Chikamoto H, Horita S, Yago R, Tanabe K, Yamaguchi Y, et al. Screening of vesicoureteral reflux in pediatric patients with kidney transplantation showing non-specific interstitial fibrosis and tubular atrophy with interstitial Tamm-Horsfall protein deposits in protocol allograft biopsy. Clin Transplant. 2009;23(Suppl 20):2-5.

17 Salvatierra O Jr, Singh T, Shifrin R, Conley S, Alexander S, Tanney D, et al. Successful transplantation of adult-sized kidneys into infants requires maintenance of high aortic blood flow. Transplantation. Oct 15 1998;66(7): 819-23.

18 Naesens M, Kambham N, Concepcion W, Salvatierra O Jr, Sarwal M. The evolution of nonimmune histological injury and its clinical relevance in adult-sized kidney grafts in pediatric recipients. Am J Transplant. 2007; 7(11):2504-14.

19 Murray BM, Paller MS, Ferris TF. Effect of cyclosporine administration on renal hemodynamics in conscious rats. Kidney Int. 1985; 28(5):767-74. 